\title{
A new Java-based application in solar physics
}

\author{
A. Sarsembayeva (iD ${ }_{1, *}{ }^{,}$F. Belisarova ${ }^{1}$, M. Odsuren ${ }^{2}$ and A. Sarsembay ${ }^{3}$ \\ ${ }^{1}$ Department of Physics and Technology, Al-Farabi Kazakh National University, \\ 71, al-Farabi Ave., 050040, Almaty, Kazakhstan \\ ${ }^{2}$ School of Engineering and Applied Sciences, National University of Mongolia, \\ Ikh surguuliin gudamj 3, 14201, Ulaanbaatar, Mongolia \\ ${ }^{3}$ School-Lyceum №250 named after T.Komekbayev, 19A, Balky bazar Str., 120500, \\ Karmakchi, Kyzylorda, Kazakhstan \\ e-mail: "sarsembaeva.a@kaznu.kz
}

\begin{abstract}
Solar-based flares are one of the most impressive energetic events in the solar atmosphere. Given their part of job in the solar corona's energy balance and their function playing important role in the space weather, numerous observations researched the release of energy and induction of solar flares, focusing on the solar active. National solar observatories are providing the overall network with a wealth of data, covering extensive time ranges (e.g. Solar and Heliospheric Observatory, SOHO), numerous perspectives (Solar Terrestrial Relations Observatory, STEREO), and returning a lot of information (Solar Dynamics Observatory, SDO). Specifically, the huge amount of SDO data are accessible only from a few repositories, such type of data as a full-cadence data or full-disk of scientific concern are hard to download, because of their size. To address these difficulties we have begun to build up a new solar application using the Java programming language for a stand-alone application type (GUI).
\end{abstract}

Key words: temperature, EM, reconnection rate, Java.

PACS number(s): 96.60.-j; 96.60.Iv; 96.60.qe

\section{Introduction}

Solar flares are powerful explosions of radiation, while coronal mass ejections are enormous clouds of solar material and magnetic fields that eject from the Sun at high speeds. Damaging emission from a solar flare cannot go through the atmosphere of the Earth and affect physically humans, but when they are sufficiently enough they can disturb the Earth's atmosphere in the layer where GPS and communications signals travel [1].

Solar burst, on the other way, directly influence to the Earth's ionosphere and radio communications on the ground, and furthermore spread of energetic particles into space. Hense, for comprehension and prediction of space weather and the solar flares impact on the Earth, a comprehension of coronal mass ejections and solar flares is required.

Solar and stellar flares have been studied using both ground and space-based investigation [2-3]. Currently, using GOES and soft X-ray data of Yohkoh Bragg crystal spectrometer, by [4] proposed between's the peak temperature of solar flares and their volume emission measure a exceptional correlation, where $n$ is the electron number density and $V$ is the volume. Shimizu [5] found a comparative connection in microflares observed by the Yohkoh soft X-ray telescope. Feldman, Laming, \& Doschek [4] show that this relationship can also be effectively extrapolated to the instanse of stellar $\mathrm{X}$-ray flares.

Figure 1 shows the observed emission measure and temperature relationship, based on solar flare data by Feldman et al. ([4]; higher hatched area), solar microflare data by Shimizu ([5]; lower hatched area), and four stellar flares by [4] (asterisks). All of the four stellar flares appear to lie on the correlation between's flare temperature and emission measure, including solar micro flares as well as stellar flares [6].

Figure 1 shows the observed ratio between the solar and stellar flares emission measure and as well as their temperatures. The emission measure and temperature's correlation given by the equation (1) is plotted as solid lines with the values of magnetic field strength $B=15,50,150 \mathrm{G}$ in the case of $n_{0}=10^{9} \mathrm{~cm}^{-3}$. Figure 1 illustrates that the observed ratio line coincide to the magnetic field 
strength's line with the $30-150 \mathrm{G}$ and for solar and stellar flares coronal magnetic field strength is evaluated to be about 40-300G. In addition, we can plot the relation between the emission measure and temperature for constant solar flare loop length from the equations (1) and (2) if we exclude the magnetic field strength, which is additionally in Figure 1 appeared in as dash-dotted lines. It is known that the characteristic size of a solar micro flaring loop is $10^{8}$ $-10^{9} \mathrm{~cm}$ and the characteristic size of a solar flaring loop is about $10^{9}-10^{10} \mathrm{~cm}$. These values completely reliable with observations [6].

The primary reason for developed Java-based solar application is to accommodate solar activity near real-time information and definition of the solar parameters. This Java application open to a wide range of users. Cross-platform application was created by following 3 standards: intuitive, simplicity and user-friendliness. The combination of these 3 standards contributes to improving the usability.

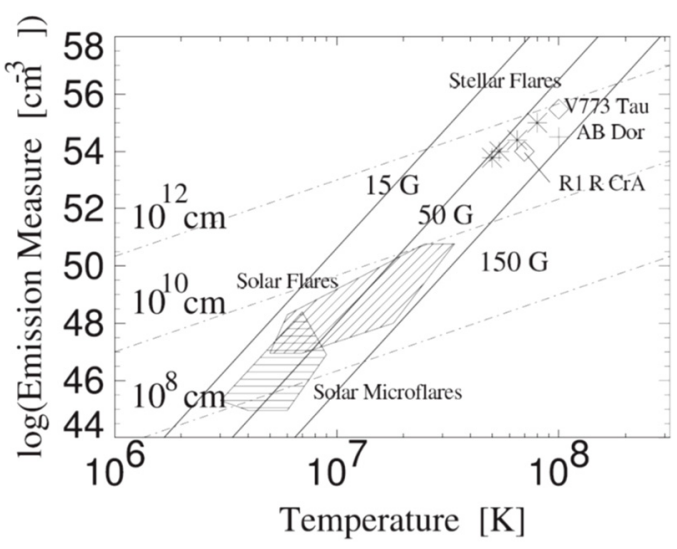

Figure 1 - The solar and stellar flares temperature and emission measure correlation [7].

\section{User interface and external tools}

In order to establish an OS independent application, Java was selected as a programming language. Java is an OS independent platform and distributed free of charge. Graphical UIs simplify use of PCs by displaying data in a way that allows rapid assimilation and manipulation. The standards of UI design, for example, flexibility and efficiency are intended to improve the quality of UI design and structure. A good GUI configuration does not require to memorize the steps needed to perform an action for program users. Taking the aforementioned facts into account, a stand-alone type application is being developed. This application is entirely written using the Swing and SwingX library (Java programming language).

The objective of this work is to accomplish better system usability through design decisions personalized in the program architecture. Advanced features implemented in program can allow users to save their time. The efficiency of application directly depends from well-designed user-interface and its functionality. The implementation of such features over-simplifies the problem and provides little guidance for the user interface designer. Therefore usage of advanced components of Java gives great opportunity to users to achieve easiness and efficiency during the compilation time. Usability is critical for the success of program and should never be overlooked. Good usability can improve the performance of program.

Figure 2 shows the GUIs main window which is partitioned into four areas. At the top of the application located a menu bar which is used to perform basic and common operations. This includes the following menus: 'File', 'Edit'. Between the menu bar and the content menu is a toolbar which provides three very frequently used controls: 'OPEN', 'EDIT', 'COPY', 'PASTE', 'SAVE', 'SELECT', 'CLEAR', and a commaseparated values file - 'CSV', which allows data to be saved in a tabular format and 'GRAPH' is a visual plotting of the relationship between variable quantities. The left panel is used to display content menu. Content menu consists 'Temperature', 'Emission measure', 'Particle acceleration', and 'Energy release' which contains two nodes 'Sweet Parker model', 'Petschek model'.

Figure 3 illustrates the layout window of Temperature and Emission measure panel. All panels offers a list of panels to get an input text value.

The efficiency of stand-alone type application directly depends on well-designed content and its functionality. A new solar application was developed using Java language. The main motivation was the need for a platform-independent language, that could be accessible in various operation systems. The primary purpose of solar application is to provide advanced functionality for the users. A solar application was designed for both beginners and expert users to allow users to save their time by using advanced features of the program. During the development of new a solar application we followed 3 key principles:

- Intuitive (no need to invest time into learning): 
You shouldn't have to read a manual to become more productive;

$\checkmark \quad$ Clean and intuitive user-interface.

- Simplicity (the quality of being easy to understand):

$\checkmark$ The Toolbar is the most important design element on an editor development because it is a singular tool that gives users a sense of orientation.

- User-friendliness (the quality of being functional):

$\checkmark$ Collapsible/expandable item;

$\checkmark$ Add/remove buttons;

$\checkmark \quad$ Filterable suggestion field;

$\checkmark$ Dynamic suggestion field.

The advanced functionalities presently used to achieve the user-friendliness are briefly described below:

- Collapsible/expandable item
Collapsible/expandable function was implemented to simplify the tasks and decisions by creating a visual representation of a user interface. This function can reduce complexity for users.

- Add/remove buttons

Similar to the concept of collapsible/expandable items, the number of input areas must be minimum at first. These areas can be added/removed interactively by buttons.

- Filterable suggestion field

Filterable suggestion field aims to save users time and allows to users to avoid the mistakes.

- Dynamic suggestion field

The main function of a dynamic suggestion fields is to make data input easier and more reliable. For example, the user chooses an input from one list, which restricts the related contents of another list.

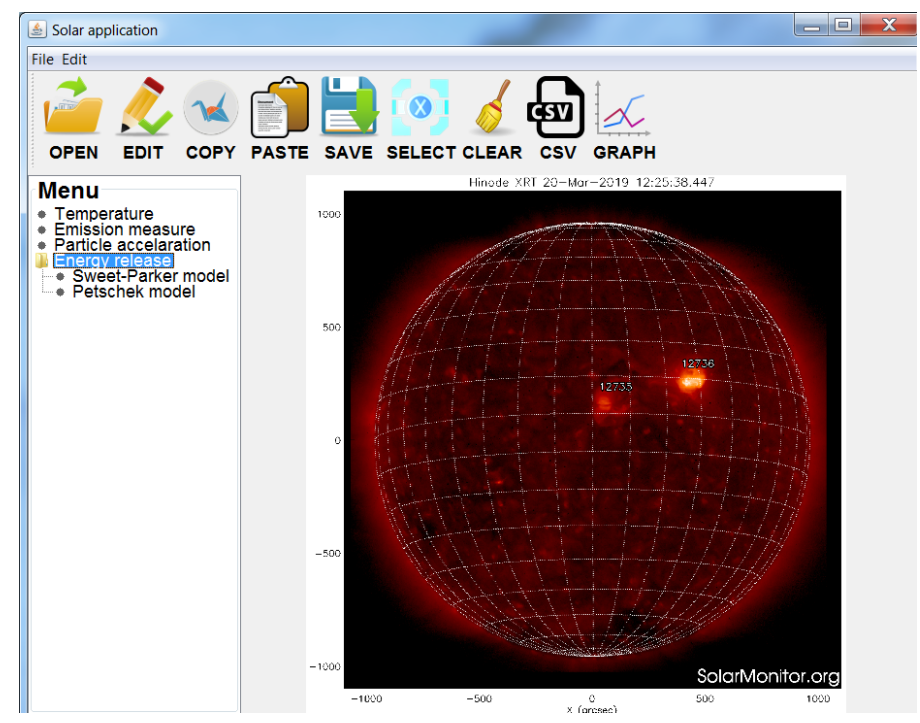

Figure 2 - The layout of main window of the Java application

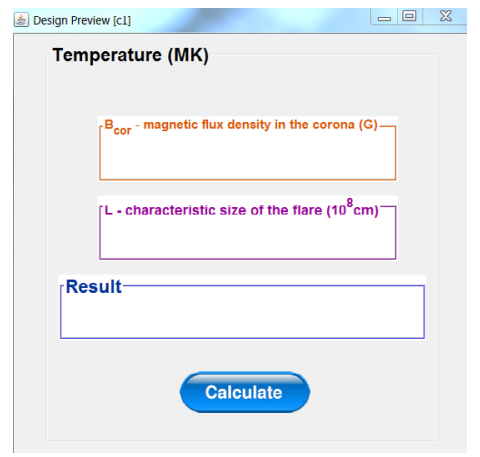

a)

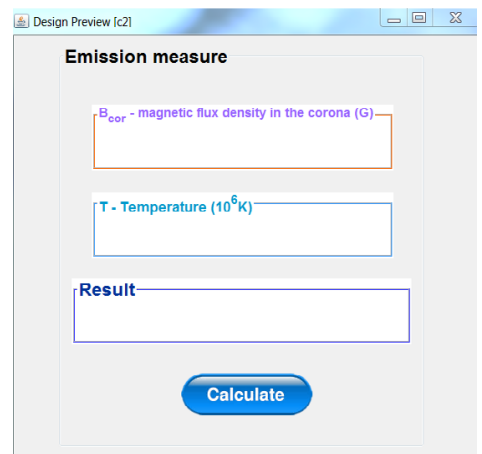

b)

Figure $3-$ a) The layout of Temperature panel.

b) The layout of Emission measure panel. 
Supported platforms: since Microsoft Windows operating systems are the most widely used computer platforms, execution of application on Microsoft Windows has been considered with a priority. The program has been tested on Windows $\mathrm{XP}, 7$ and 10. Application execution on the GNU/Linux operating system has not been tested very often, but we confirmed it runs under Ubuntu 14.04.3 LTS. Application has not yet been tested on Mac OS.

\section{Method}

For quantitative physical comprehension of processes in the atmosphere of the Sun, the X-ray fluxes are in a limited of use. However, they affect the emission measure and temperature of the plasma that delivers the soft X-rays, and these physical quantities are vital: from them, the energetics of solar flares and other energy releases can be deduced.

[4] presented that there is a ratio between solar flare emission measure and temperature for solar flares as well as for some of stellar flares. [7] continued this ratio between emission measure and temperature and applied it for solar micro flares, TTauri star flares, and protostellar flares. It is outstanding that the relationship holds in a very wide range, $4 \times 10^{6} \mathrm{~K}<T<10^{8} \mathrm{~K}$ and $10^{45} \mathrm{~cm}^{-3}<$ $E M<10^{56} \mathrm{~cm}^{-3}$. Shibata and Yokoyama [7] then could find that this universal correlation can be interpreted by the simple scaling law (see Figure 1),

$$
E M \simeq 10^{48} \mathrm{~cm}^{-3}\left(\frac{B}{50 \mathrm{G}}\right)^{-5}\left(\frac{n_{0}}{10^{9} \mathrm{~cm}^{-3}}\right)^{3 / 2}\left(\frac{T}{10^{7} \mathrm{~K}}\right)^{17 / 2},
$$

The temperature were obtained in SunPY using the methods of White et al. [10-11] who used the CHIANTI atomic physics database to model the response of the ratio of the short (0.5-4 angstrom) to long (1-8 angstrom) channels of the XRSs onboard various GOES satellites [12].

Yokoyama \& Shibata [8] performed magnetohydrodynamic numerical simulations of the reconnection, including both heat conduction and chromospheric evaporation and found a simple scaling relation:

$$
T=10^{7} K\left(\frac{B}{50 G}\right)^{6 / 7}\left(\frac{n_{0}}{10^{9} \mathrm{~cm}^{-3}}\right)^{-1 / 7}\left(\frac{L}{10^{9} \mathrm{~K}}\right)^{2 / 7},
$$

Evaluated convective electric field related to the magnetic reconnection rate, which conceivably during a solar flare takes a significant part in charged particle acceleration [6]:

$$
E=3 \times 10^{3}\left(\frac{M_{A}}{0.1}\right)\left(\frac{B}{100 G}\right)^{2}\left(\frac{n_{j e t}}{10^{10} \mathrm{~cm}^{-3}}\right)^{-\frac{1}{2}} V m^{-1}
$$

The magnetic reconnection rate is commonly considered to play a key role in the build-up of energy in solar flares. The reconnection rate is an significant quantity, since it puts demanding constraints on the magnetic reconnection model. In spite of its significance, what defines the flares magnetic reconnection rate is still an open question. Sweet [13] and Parker's [14-15] steady reconnection model have a proposed reconnection rate value, which is equal to the $M_{A}=\left(\operatorname{Re}_{m}\right)^{-1 / 2}$, $\operatorname{Re}_{m}=\left(V_{A} L / \eta\right)$ is the magnetic Reynolds number characterized by the Alfven velocity and $\eta$ is the magnetic diffusivity $\eta \sim 10^{4}\left(\frac{T}{10^{6} \mathrm{~K}}\right)^{-3 / 2} \mathrm{~cm}^{2} \mathrm{~s}^{-1}$. Petschek [16] identified that the previous model lacks the impacts of waves and proposed his model with $M_{A} \lesssim \pi /\left[8 \ln \left(8 \operatorname{Re}_{m}\right)\right][17-21]$.

\section{Results}

The length of a stellar flaring loop $\left(10^{10}-10^{12}\right.$ $\mathrm{cm}$ ) is much larger than the characteristic size of a solar flares. This value is reliable with observations at the surface of young stars is very strong that average field strength is order kilo Gauss [9].

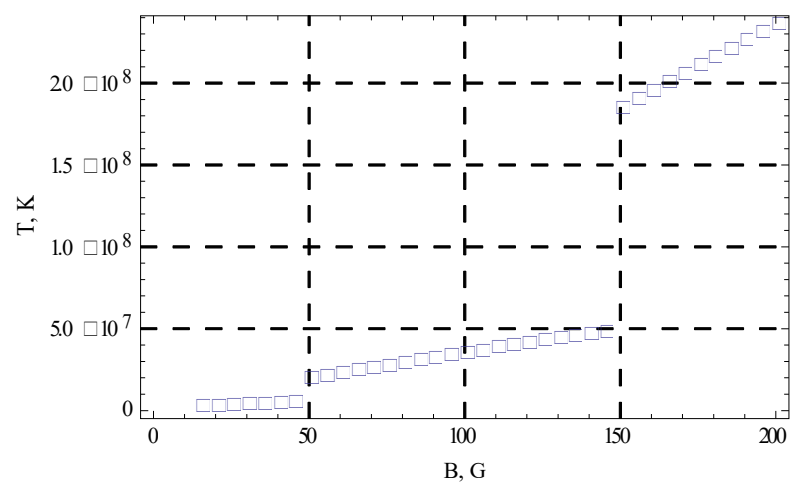

Figure 4 - The correlation between temperature and magnetic flux density of solar and stellar flares.

The motivation behind why some of stellar flares, particularly young star flares, show high temperature and exceptionally a large amount of 
total energy, which is because the characteristic size of stellar flares is much larger than the solar flares. When the length of a flaring loop have a large value, the temperature of a solar flare increases in proportion to $L^{2 / 7}$ regardless of whether the magnetic field is the equivalent, for the reason that the conduction cooling become less efficient for a longer loop [22-25]. Figure 4 shows the correlation between the temperature of solar and stellar flares and their magnetic flux density obtained using the solar application.

\section{Conclusions}

A new Java-based application is being developed by Java as a stand-alone application, which provides an environment for determination of emission measure and temperature of solar flares. A solar application also allow to users define the magnetic reconnection rate (Sweet-Parker and Petschek model). The Swing component library was used to implement the GUI. In the current stage, the design of the user interface, coding for input forms and link with utilities were implemented. A solar application was developed by following 3 principles: intuitive, simplicity and userfriendliness. The combination of these 3 principles contributes to improving the usability. Advanced features implemented in the program can allow users to save their time. Java is basically an OS independent platform, however, tests on each OS may be necessary. At present, the test was only performed in Microsoft Windows and Linux, and will be performed in Mac OS in near future.

\section{References}

1. Garcia, H. A. (2004). Forecasting meth. for occurrence and magnitude of proton storms with solar hard X rays. Space Weather, Vol. 2, S06003.

2. Svestka, Z., Cliver, E.W. (1992). Eruptive sol. flares. Lecture Notes in Physics, 399, 1.

3. Haisch, B., Strong, K.T. and Rodono, M. (1991). Flares on the Sun and other Stars. Ann. Revs. Astron. Astrophys., 29, 275.

4. Feldman, U., Laming, J.M., Doschek, G.A. (1995). The correlation of Sol. flare temp. and EM Extrapolated to the Case of Stellar Flares. Astrophys. J. Lett., 451, L79.

5. Shimizu, T. (1995). Energetics and Occurrence Rate of Active-Region transient brightenings and implications for the heating of the active-region Cor. Publ. Astron. Soc. Japan, 47, 251-263.

6. Shibata, K., Magara, T. (2011). Sol. flares: Magnetohydrodynamic Processes. Living Rev. Sol. Phys. 8, 6.

7. Shibata, K., Yokoyama, T. (1999). Origin of the Universal correl. between the flare temp. and the EM for Solar and Stellar Flares. Astrophys. J. Lett., 526, L49-L52.

8. Yokoyama, T., Shibata, K. (1998). A Two-dimensional Magnetohydrodynamic simulation of chromospheric Evaporation in a Solar Flare Based on a Mag. Reconnection Model. Astrophys. J. Lett., 494 , L113.

9. Johns-Krull, C.M., Valenti, J.A., Koresko, C. (1999). Measuring the Mag. field on the Classical T Tauri Star BP Tauri. Astrophys. J., 516, 900-915.

10. White, S.M., Thomas, R.J. \& Schwartz, R.A. (2005). Updated expressions for determining temp. and EM from goes soft X-ray measurements. Sol Phys, 227, 231.

11. Feldman, U. and Widing, K. G. (2003). Elemental abundances in the Sol. upper atmosphere derived by spectroscopic means. Space Sci Rev. 107, 665.

12. The Sunpy Community et al. (2015). SunPy-Python for sol. phys. Comput. Sci. Disc., 8, 014009.

13. Sweet P.A. (1958). Electromagnetic Phenomena in cosmical phys. Cambridge: Cambridge Univ. Press.

14. Parker E.N. (1957). Sweet's mechanism for merging mag. fields in conducting fluids.J. Geophys. Res., Vol.62, 509-520.

15. Parker E.N. (1963). The sol. flare phenomenon and theory of reconnection and annihilation of mag. fields. Astrophys. J. Supp., Vol.8, 177-211.

16. Petschek H.E. (1964). Mag. field annihilation. Physics of Solar Flares/ed. by W.N. Hess. NASA SP-50. Washington: DC, 425-439.

17. Sarsembayeva A.T., Belisarova F.B., Odsuren M., Sarsembay A.T., Kalymova Zh. (2012). Definition of reconnection rate of sol. flares registered in 2011-2012 years. Adv. Studies Theor. Phys., Vol.6, no.28, 1405-1408.

18. Sarsembayeva A.T., et al. (2019). 26 January, 2019 sol. flares diagnostics based on the soft x-ray EM. NEWS of the National Academy of Sciences of the Republic of Kazakhstan. Series of Physical and Mathematical. №2, 41-46. 
19. Nitta S. (2004). Outflow structure and reconnection rate of the self-similar evolution model of fast mag. reconnection. Astrophysical Journal, Vol.610, 1117.

20. Matthaeus W.H., Lamkin S.L. (1985). Rapid mag. reconnection caused by finite amplitude fluctuations. Phys. Fluids, Vol.28, 303.

21. Tajima T., Shibata K. (1997). Plasma astrophys. Addison- Wesley.

22. Dere K.P. (1996). The rate of mag. reconnection observed in the sol. atmosphere. ApJ., Vol.472, 864.

23. Tsuneta S. (1996). Structure and dynamics of mag. reconnection in a sol. flare. The Astrophysical Journal, Vol.456, 840-849.

24. Tsuneta S., Masuda S., Kosugi T., Sato J. (1997). Hot and superhot plasmas above an impulsive flare loop. ApJ., Vol.478, 787.

25. Isobe H., Yokoyama T., Shimojo M., Morimoto T., Kozu H., Eto S., Narukage N., Shibata K. (2002). Reconnection rate in the decay phase of a long dur. event flare on 1997 May 12. ApJ., Vol.566, 528. 